toxic $\mathrm{T}$ cells (reactive to $\mathrm{H}-2$ antigens) by the use of anti-Ia antiserum, which lyses the former, and also by anti-Ly1.1, which lyses cytotoxic cells of the CBA strain. On the other hand, the precursors of suppressor cells are usually not lysed by anti-Ia antiserum ${ }^{11}$, and thus the two cell types could still be closely related.

It is now clear, however, that the Ly-1 and Ly-2 populations belong to different lines of differentiation and are not sequential stages in a single lineage $^{12}$. Cantor and his colleagues have shown that mice depleted of $\mathrm{T}$ cells (thymectomised, irradiated and bonemarrow-reconstituted mice or " $B$ mice") and repopulated with Ly-1 T cells only were not able to perform any of the functions of $\mathrm{Ly}-2,3$ cells, even many months later; and conversely "B mice" repopulated with Ly-2,3 cells could express cytotoxicity and suppression, but not other $T$ cell functions.

There is great interest in the role of the major histocompatibility complex (MHC) in immune responses. One aspect which has attracted attention is the prohibition of cell interactions across allogeneic barriers where MHC differences are involved. This has been reported for $\mathrm{T}-\mathrm{B}$ interactions by $\mathrm{Katz}$ and colleagues ${ }^{13}$, for $T$-macrophage interactions by Rosenthal and Shevach ${ }^{14}$ and Erb and Feldmann ${ }^{15}$, and for $\mathrm{T}$ cell killing, by Zinkernagel, Blanden and Doherty $^{26}$. Several explanations have been suggested for these restrictions. One is that a molecule controlled by the MHC must be "shared" or recognised for successful interactions to occur $^{13}$. An alternative suggestion is that mixing histoincompatible cells may induce active suppression ${ }^{9,17}$. D. H. Katz (Harvard University), for example, found that suppressor cells are induced when $F_{1}$ thymocytes are primed in an irradiated parental mouse. These suppressor cells may explain the genetic restrictions on $T-B$ collaboration found in earlier experiments ${ }^{13}$. Support for this possibility was discussed by $\mathbf{H}$. Cantor and R. K. Gershon (Yale University) who have shown that removal of Ly-2,3 cell populations (which contain suppressor cells) permits the residual $T$ cells to collaborate with histoincompatible $\mathbf{B}$ cells in the primary response in vitro to sheep red cells. Independently, Dutton and his colleagues ${ }^{17}$ have obtained similar results using anti-Ly antisera and cell separation techniques, indicating that allogeneic effects can suppress interactions between histaincompatible $\mathrm{T}$ and $\mathrm{B}$ cells. Thus, there is no doubt that suppression explains some examples of the failure of histoincompatible $\mathbf{T}$ and $\mathbf{B}$ cells to interact. Whether all such failures can be attributed to suppression is not yet clear.

\title{
EGF and viral transformation
}

\author{
from Robin A. Weiss
}

EPIDERMAL growth factor (EGF) was (KNRK) and by Rous sarcoma virus discovered by Stanley Cohen (J. biol. (XC) apparently lacked EGF receptors. Chem., 237, 1555; 1962) when he was Reduction in the binding of other purifying nerve growth factor from hormones to transformed cells has also the submaxillary salivary gland of male been reported, for example, in a recent mice. Cohen noted that the factor study of insulin receptors on BALB/ stimulated the proliferation and dif- $3 \mathrm{~T} 3$ cells by Thomopoulous, Roth, ferentiation of the epidermis, as re- Lovelace and Pastan (Cell, 8, 417; flected by precocious opening of the 1976). The binding of insulin varied eyelids and eruption of the incisors in with the level of proliferation in cell baby mice. Since that time it has been population and the reduction seen in Cohen's remarkable achievement to transformed clones was not specific to purify and sequence murine EGF the transforming agent. However, the (Cohen and Savage, Recent Progress in presence of EGF receptors appears to Hormone Research, 30, 551; 1974). It be more specific. In this issue of is a single-chain polypeptide containing Nature (page 26), Cohen, in col53 amino-acid residues with a mole- laboration with Todaro and De Larco, cular weight of 6,045 . The molecule has reports additional data on the binding been well conserved during evolution of EGF to the same cell types transand human EGF extracted from urine formed by different tumour viruses. shows only minor differences in amino- For example, the A31 clone of mouse acid sequence to murine EGF. Re- BALB/3T3 cells was transformed by cently it has become evident that EGF SV40, two strains of mouse sarcoma will stimulate proliferation of a variety virus (MSV) and Rous sarcoma virus of both epithelial and fibroblastic cells (RSV). Only the MSV-transformed in culture. Its specific mitogenic action subclones failed to bind EGF. This on non-proliferating cells in culture remarkable specificity of binding was provides a much more convenient and true for transformed subclones of quantitative assay than examining eye- other types of cell such as Swiss 3T3, lid opening in vivo.

NRK and Mink lung. In each case,

Last year Cohen's group showed that only the subclones transformed by cells in culture which respond to the MSV or by feline sarcoma virus failed mitogenic action of EGF possess spe- to bind EGF.

cific receptors for EGF on the cell Solely on the basis of the specific surface (Carpenter, Lembach, Mor- failure to bind EGF, the authors posrison and Cohen, J. biol Chem., 250, tulate that MSV might transform cells 4297 ; 1975). EGF receptors were by coding for an EGF-like molecule in assayed by measuring the binding of part of its sarcoma-specific genetic ${ }^{125}$ I-labelled EGF to the cell surface. sequences. This EGF analogue would Cells of many species bound EGF, but then block the receptors and stimulate certain types, including a human lym- the cells to grow. This will hardly prophoblastoid line (NC-37) and rat cells vide the whole explanation of viral transformed by mouse sarcoma virus oncogenesis, but it can be tested.

It now seems clear from the reports of several workers that suppression is mediated by products of the $I$ region. $\Gamma$ cell suppression in mice, for example, is mediated by an antigen-specific $T$ cell factor with a molecular weight of about 50,000 and which does not operate across $\mathrm{H}-2$ barriers $^{18,19}$ (T. Tada, Chiba University). This factor is absorbed by antisera reactive to products of the I-J subregion, which also seems to code for the receptor for the suppressive factor. The cells carrying the receptors (sometimes known in this context as acceptors) were found not to be helper $T$ cells, but nylon-wool-adherent $T$ cells which could be killed by anti-I-J serum and complement ${ }^{18,19}$. Allotype specific suppressor $\mathrm{T}$ cells can also be killed by antiserum containing antibody to I-J region products ${ }^{20-12}$ (L. Herzenberg and D. B. Murphy, Stanford University).

Two generalisations seem to be possible on the basis of this and other work. First, that the I region is functionally specialised, the I-J subregion being involved in suppression and the $\mathrm{I}-\mathrm{A}$ subregion in macrophage or $\mathrm{T}$-cell helper functions. Second, the products of the I region act on target cells through receptors controlled by the same I subregion ${ }^{23,24}$.

M. Taniguchi (Chiba University) discussed studies performed with Tada on a factor obtained from lysed antigenprimed spleen or thymus cells which augmented IgG antibody responses, provided that factor donor and recipient (in culture) were identical in the I-A region. This factor seems to be different from the helper factor described by 